\title{
Bestellung und Abberufung von Betriebsbeauftragten- am Beispiel von Universitätskliniken und Krankenhäusern
}

\section{Einführung}

Die Bedeutung des Betriebsbeauftragten ist in den letzten Jahren immer stärker gewachsen. Die Rechtswissenschaft hat sich bislang jedoch nur zögerlich mit seiner rechtlichen Stellung befaßt. Fast scheint es, als sei das Interesse in dem Maße geschwunden, je mehr die Beauftragtenregelungen in umwelt- und arbeitsrechtliche Fachgesetze integriert wurden. Dabei fällt auf, daß insbesondere die Frage nach der Mitbestimmung bei der Bestellung und Abberufung des Betriebsbeauftragten weitgehend ausgespart blieb. Das muß um so mehr verwundern, als eine betriebshierarchische Positionierung des Beauftragten zwischen Personalvertretung und Betriebsleitung für dessen Selbstverständnis wie auch für eine wirksame Aufgabenerfüllung von herausragender Bedeutung ist. Im folgenden soll daher der mitbestimmungsrechtliche Komplex näher untersucht werden, wobei das Thema zunächst in zweierlei Hinsicht zu beschränken ist. Zunächst gilt es, die personellen Entscheidungen der Einsetzung und Abberufung von Betriebsbeauftragten vor dem Hintergrund einer die technischen Risiken kumulierenden Lebenswelt aufzuwerfen, wo sie in der Praxis nicht selten in den Sog mitbestimmungsrechtlichen Disputs zwischen öffentlichem Arbeitgeber und Personalrat hineingezogen werden: gemeint sind staatliche Krankenhäuser und Universitätskliniken. Diesem Anwendungsbereich entsprechend erfolgt zudem eine Verengung der Rechtsfragen auf das Betriebsorganisationsrecht des öffentlichen Dienstes. Die Einbindung der mitbestimmungsrechtlichen Frage in den konkreten Bezugsrahmen eines Subsystems der Daseinsvorsorge hat den Vorteil, daß die Multidimensionalität der Rechtsfigur des Betriebsbeauftragten erkennbar wird und ermöglicht eine übergreifende rechtspolitische Diskussion der zukünftigen Ausgestaltung dieses Instruments indirekter Verhaltenssteuerung (V). Zuvor soll jedoch ein kurzer Blick auf das Rechtsinstitut des Betriebsbeauftragten geworfen werden (II). Im Anschluß daran erfolgt eine Darstellung der diversen Beauftragtenregelungen im Krankenhausbetrieb (III). Schließlich geht es um die Prüfung verschiedener Mitbestimmungstatbestände der Personalvertretung bei der Bestellung und Abberufung der verschiedenen Beauftragten (IV).

\section{Stellung und Funktion des Betriebsbeauftragten}

In den letzten Jahren vollzieht sich im Umweltrecht ein schrittweiser Flexibilisierungsprozeß, dessen Auswirkungen auch das allgemeine Verwaltungsrecht nicht unberührt ließ und es vermutlich auch zukünftig noch stark beeinflussen wird.' Im Zuge dieser Entwicklung wird die herkömmliche Eingriffsverwaltung als ein statisches, an administrativer Außensteuerung ausgerichtetes Regelungskonzept zuneh-

I Hierzu vgl. den Entwurf eines Umweltgesetzbuches: Kloepfer/Rehbinder/Schmidt-Aßmann/Kunig, Umweltgesetzbuch-Allgemeiner Tel, sechstes Kapitel; zu neuen Entwicklungen im Verwaltungsrecht, Hoffmann-Riem, Ermoglichung von Flexibılitat und Innovationsoffenheit im Verwaltungsrecht, in: Flexibilıtat und Innovationsoffenheit im Verwaltungsrecht, Baden-Baden 1994, hrsg. von Hoffmann-Riem und Schmidt-Aßmann, S. 31 ff.; vgl. auch den Berıcht des Umweltsachverstandigenrats, BT-Drucks. 1 2/6995, S. $144 \mathrm{f}$. 
mend vom gesetzgeberischen Leitbild der innerbetrieblichen Steuerung flankiert: Genannt seien in diesem Zusammenhang etwa die Verpflichtung zur umweltsichernden Betriebsorganisation und betrieblichen Eigenüberwachung ${ }^{2}$, die Ermittlungsverantwortlichkeit des Vorhabenträgers im Rahmen des UVP-Gesetzes, die freiwillige Durchführung sogenannter Öko-Audits und schließlich die gesetzliche Pflicht zur Bestellung von Betriebsbeauftragten.

Die Einrichtung des Betriebsbeauftragten verdankt ihre Existenz jedoch nicht erst innovativen Tendenzen des neueren Umweltrechts, sondern wurde vor allem auf dem Gebiet des Arbeitsschutzrechts legislativ umgesetzt. Die Idee, dieses Konzept auch dem Umweltschutzrecht zu inkorporieren, entstammt hingegen den Anfängen der Umweltpolitik zu Beginn der 7oer Jahre. ${ }^{3}$ Die Funktion des Betriebsbeauftragten Jäßt sich im Hinblick auf die mittlerweile außerordentlich breite Palette der fachgesetzlich normierten Anwendungsfelder zunächst einmal ganz pauschal als Mitwirkung zur innerbetrieblichen Gefahrenabwehr umschreiben. Damit sind aber nicht nur die Gefahren für den Betrieb und seine Mitarbeiter selbst gemeint, sondern auch die aus betriebsimmanenten technischen Risiken für die Außenwelt resultierenden Gefährdungspotentiale.

Allerdings unterscheiden sich die Mittel, deren sich Betriebsbeauftragte zur Erfüllung ihrer Aufgaben zu bedienen haben, von denen auf dem Gebiet der Gefahrenabwehr wesentlich. Während ordnungsbehördliches Verwaltungshandeln grundsätzlich subordinativen Charakter hat, obliegt es dem Betriebsbeauftragten in erster Linie durch Konsensbildung, weniger durch Anordnung und Überwachung, die Sicherheit im und außerhalb des Betriebs zu gewährleisten. Neben die Überwachungsfunktion treten deshalb in größerem Umfang auch Beratungs- und Innovationsfunktionen, so daß man gemäß der gesetzgeberischen Leitidee den Betriebsbeauftragten als Ausprägung des Prinzips eigenverantwortlicher Selbststeuerung im Verwaltungsrecht ansehen muß.

Diese grobmaschige Beschreibung kann nicht darüber hinwegtäuschen, daß die Figur des Betriebsbeauftragten im Koordinatenkreuz zwischen quasi öffentlich-rechtlicher Beleihung ${ }^{4}$ einerseits und dem beschäftigungsrechtlichen Grundverhältnis andererseits nicht ohne Probleme innerhalb des Rechtssystems zu verorten ist. Die daran anknüpfenden Probleme führen jedoch über den hier zu erörternden Fragenbereich hinaus und müssen einer grundsätzlichen, die rechtliche Stellung des Betriebsbeauftragten klärenden Monographie vorbehalten bleiben.

\section{Gesetzliche Bestellungserfordernisse für Krankenhäuser und Universitätskliniken}

Der Arbeitsplatz Krankenhaus birgt eine Reihe von Gefahrenquellen nicht nur für die Binnenstruktur, wozu etwa Personal, Patienten und Publikumsverkehr zu zählen sind, sondern auch - freilich eingeschränkt - für die Außenwelt: man denke etwa an mit Krankheitserregern kontaminierten Krankenhausabfall oder die aus dem Umgang mit radioaktivem Klinikmüll erwachsenden Risiken. Die nicht ordnungsgemäße Entsorgung gebrauchter Spritzen beispielsweise stellt eine erhebliche Gesund-

2 Etwa in $\$ \$ 26,27,29,52$ a BImSchG; $\$ \$ 19,121$ WHG.

$3 \mathrm{Vgl}$. Rehbinder/Burgbacher/Knieper, Ein Betriebsbeauftragter fur Umweltschutz?, Berlin 1972, mit einer Zusammenfassung der damals bestehenden gesetzlichen Ansatze zur Beauftragtenregelung, S. I off.; Rehbinder weıst an anderer Stelle darauf hın, daß die Idee des Betrıebsbeauftragten sich bis zum Sicherheitsbeauftragten des Preußischen Berggesetzes von 1865 z zuruckverfolgen laßt, Reflexives Recht und Praxis Der Betriebesbeauftragte fur Umweltschutz als Beispiel, Jahrbuch f. Rechtssoziologie 1988, S. 117. 4 Kritisch zur Beleihungsfigur, Steiner, Technische Kontrolle im privaten Bereich, DVBI. 1987, S. 1 I 38. 
heitsgefährdung der mit der Müllbeseitigung beschäftigten Personen dar.s Um das gesamte Spektrum betriebsspezifischer Risiken möglichst eigenverantwortlich unter Kontrolle zu bringen, finden sich eine Reihe von gesetzlichen Bestimmungen über die Einsetzung von Betriebsbeauftragten. Diese lassen sich im Hinblick auf ihre jeweilige Schutzrichtung in verschiedene Kategorien einteilen.

\section{Die Beauftragtenregeln für die Arbeitssicberbeit}

Nach $\$ 719$ I RVO hat der Unternehmer eines Betriebs mit mehr als 20 Beschäftigten einen oder mehrere Sicherheitsbeauftragte zu bestellen. Die Aufgabe der Sicherheitsbeauftragten besteht nach $\$ 719$ II RVO darin, den Unternehmer bei der Durchführung des Unfallschutzes zu unterstützen, insbesondere sich von dem Vorhandensein und der ordnungsgemäßen Benutzung der vorgeschriebenen Schutzvorrichtungen fortlaufend zu überzeugen.

Eine weitere arbeitsrechtliche Beauftragtenregelung enthält das Arbeitssicherheitsgesetz. Danach hat der Arbeitgeber Fachkräfte für Arbeitssicherheit zu bestellen, die ihn beim Arbeitsschutz und der Unfallverhütung unterstützen. Der Aufgabenbereich dieser Fachkräfte geht über den von $\$ 719$ RVO gesteckten Rahmen hinaus. $\$ 6$ ASiG enthält neben einem detaillierten Aufgabenkatalog auch die grundlegende Verpflichtung, den Arbeitgeber bei der menschengerechten Gestaltung der Arbeit zu unterstützen. Nach $\$ I $6 \mathrm{ASiG}$ ist ein gleichwertiger arbeitsmedizinischer und sicherheitstechnischer Arbeitsschutz auch in Verwaltungen und Betrieben des öffentlichen Dienstes zu gewährleisten; mithin gilt diese Vorschrift auch für die Beschäftigungsverhältnisse in staatlichen Krankenhäusern und Universitätskliniken.

\section{Der Beauftragte zum Schutz vor medizintypischen Risiken}

Besondere Bedeutung kommt den typischen Gefährdungen, die sich aus dem Einsatz der medizintechnischen Geräte stellen, zu. Hierunter sind sowohl zu diagnostischen Zwecken eingesetzte Röntgengeräte, aber auch die bei der Ausübung der Heilkunde benötigten diversen Geräte der Nuklearmedizin zu verstehen. ${ }^{6}$

Die Verpflichtung zur Bestellung eines Strahlenschutzbeauftragten nach der Röntgenschutzverordnung folgt aus $\mathbb{I}_{3}$ I RöVO. Danach hat derjenige, der eine Röntgeneinrichtung oder einen genehmigungspflichtigen Störstrahler betreibt, einen oder mehrere Strahlenschutzbeauftragte zu bestellen. Neben einer Unterrichtungsund Beratungsfunktion hat der Strahlenschutzbeauftragte nach $\$$ Is II Nr. I und Nr. 2 RöVO dafür zu sorgen, daß die den Strahlenschutzverantwortlichen treffenden umfänglichen Schutzvorschriften eingehalten werden. Zudem hat er darüber zu wachen, daß die Bestimmungen über Genehmigung oder Bauartzulassung und die von der Behörde erlassenen Anordnungen und Auflagen eingehalten werden.

Ähnlich konzipiert sind die Pflichten des Strahlenbeauftragten nach der Strahlenschutzverordnung. Auch ihm obliegt es, innerhalb des innerbetrieblichen Entschei-

s Laut Verwaltung des Gottinger Universitatsklinikums sind bei den Beschaftigten der Mullbeseitigung ein halbes Dutzend Stıchverletzungen durch Kanulen im Jahr zu verzeichnen, Gottınger Tageblatt vom 27. 10. 1994; entsprechendes meldete das Hamburger Abendblatt in der Ausgabe vom 6. 12.1994 unter dem Titel »Die todliche Gefahr ıst uberall«, Reinıgungs" und Pflegepersonal sowie Arzte an Hamburger Krankenhausern seien seiner Vielzahl von Belastungen ausgesetzt ", insbesondere die Haufigkeit von Stichverletzungen durch gebrauchte Spritzen, Kanulen oder verschmutzte Scherben bei den Reingungskräften seı alarmierend. Von Gefahrdungen bei Mitarbeitern durch Radioaktivitat im universitaren Bereich berichtete der Spiegel, Nr. 20 v. I5. 5. 1995 , S. 78

6 Zur Systematik der Abgrenzung zwischen Strahlenschutz- und RontgenVO siehe die Kommentierung von Kramer/Zerlett, Strahlenschutzverordnung, 3. Aufl., Stuttgart 1990, zu $\$$ i StrahlSchVO, S. I 8 f. 
dungsbereiches gem. $\S_{3}$ II Nr. 1 und 2 StrahlSchVO die Strahlenschutzgrundsätze sowie die durch die Strahlenschutzverordnung angeordneten Pflichten des Strahlenschutzverantwortlichen zu überwachen. Darüber hinaus hat er gem. $\ 3_{1}$ IV StrahlSchVO dafür zu sorgen, daß bei Gefahren für Leben, Gesundheit oder bedeutende Sachwerte geeignete Maßnahmen zur unverzüglichen Gefahrenabwehr getroffen werden. Durch diese recht weitgehende Ermächtigung erhält das Profil des Betriebsbeauftragten im Strahlenschutzrecht deutlichere ordnungsbehördliche Konturen; hier ist die Funktion der Abwehr innerbetrieblicher Gefahren verknüpft mit der Befugnis, diese im konkreten Fall auch durchzusetzen. ${ }^{7}$

Schließlich ordnet auch das Medizinproduktegesetz vom 2.8.1994 in $\$ 3$ I I MedProdG eine Pflicht zur Bestellung eines Sicherheitsbeauftragten für Medizinprodukte an. Adressaten dieser Regelung sind Hersteller von Medizinprodukten sowie ihnen nach $\$ 7$ gleichgestellte Personen, die Medizinprodukte in den Verkehr bringen. Nach der extensiven gesetzlichen Definition des $\mathbb{3}$ Nr. I 2 MedProdG fällt darunter jede Abgabe von Medizinprodukten an andere, so daß die Notwendigkeit zur Bestellung von Medizinbeauftragten auch für Krankenhäuser besteht. ${ }^{9}$

\section{Der Beauftragte nach dem Abfallgesetz}

Neben dem Betriebsbeauftragten für Immisionsschutz und für Wasserreinhaltung stellt der Abfallbeauftragte den dritten, Mitte der 7oer Jahre eingeführten Beauftragten in einem zentralen Bereich des Umweltrechts dar. ${ }^{10}$

Nach $₫ 11$ a I AbfG haben Betreiber ortsfester Abfallentsorgungsanlagen sowie Betreiber von Anlagen, in denen regelmäßig gesundheits- und umweltrelevante Abfälle nach $\$ 2$ II AbfG anfallen, einen oder mehrere Betriebsbeauftragte für Abfall zu bestellen.

Die Verpflichtung zur Bestellung von Abfallbeauftragten folgt für Krankenhäuser und Kliniken aus $§ 1$ II Nr. 7 der aufgrund des $₫$ I I a I S. 3 AbfG erlassenen Verordnung über Betriebsbeaufragte für Abfall. Neben einer Überwachungsfunktion treffen ihn auch Informations-, Berichts- und Beratungspflichten gegenüber dem Betreiber wie auch gegenüber den Betriebsangehörigen gem. \$ I I b I Nr. 3, II AbfG. Zudem erfüllt er eine spezifisch umweltrechtliche Initiativfunktion sowohl bei Verfolgung des Ziels der Abfallvermeidung als auch bei der Verbesserung der Verfahren zur Abfallbeseitigung nach $\$ 11$ b IV Nr. 4 und Nr. s AbfG. Seine Überwachungspflichten sind recht weit gesteckt, ohne daß er durch eine Ermächtigungsgrundlage zur Gefahrenabwehr quasi-polizeiliche Funktionen erhält. Neben der Überwachung der Einhaltung abfallrechtlicher Regelungen, insbesondere durch regelmäßige Kontrolle der Betriebsstätten, obliegt ihm die Überwachung der Abfälle von ihrer Entstehung bis zu ihrer Entsorgung. ${ }^{11}$

\footnotetext{
7 Die Sonderstellung des Strahlenschutzbeauftragten wird thematisiert u.a. von Kloepfer, Umweltrecht, Munchen 1989, S. I 52 .

8 BGBl. I, S. 1963.

9 Zum Begriff Medizinprodukte vgl. $\$ 3$ Nr. I-7 MedProdG, kritisch zum weiten Anwendungsbereich dieser Regelungen Deutsch, Das Gesetz uber Medizinprodukte von 1994, NJW 1995, S. 753.

10 Regelungen uber den Wasserschuczbeauftragten finden sich in $\$ \$ 21$ aff. WHG und fur den Immissionsschutzbeauftragten in $\$ \$ s 3 \mathrm{ff}$. BImSchG, vgl. auch Kahl, Die neuen Aufgaben und Befugnisse des Betriebsbeauftragten nach Wasser-, Immıssionsschutz- und Abfallrecht, Bad Kissingen, 1978 sowie Sander, Rechtsstellung und Rechtsschutz des Betriebsbeauftragten fur Gewasserschucz aus der Sicht der Industrie, NuR 1985, S. 47 ff. und Roth, Der Betriebsbeauftragte fur Immissionsschutz, Frankfurt 1979.

1 I Naher zum Abfallbeauftragten, Versteyl/Hoyer, Der Betriebsbeauftragte fur Abfall: Einfuhrung in das Abfallrecht und Lettfaden für die behördliche Praxis, Boblingen 1992.
} 
Vor allem in Universitätskliniken dürfte sich im Rahmen der umfangreichen Forschungsaktivitäten regelmäßig das Erfordernis einer Bestellung weiterer Betriebsbeauftragter nach dem Gentechnik- und dem Tierschutzgesetz ergeben.

4.I. Das Gentechnikgesetz enthält in $\$ 6$ IV Gentech G für denjenigen, der gentechnische Arbeiten oder Freisetzungen durchführt, die Verpflichtung, Beauftragte für biologische Sicherheit zu bestellen. Nach $\$ I 8 GenTSVO zählen zu dem Aufgabenbereich des Beauftragten u. a. die Überwachung der gesetzlichen Verpflichtungen des Projektleiters, insbesondere durch regelmäßige Kontrolle der Laboratorien bzw. Produktionsstätten sowie Mitteilungs- und Beratungspflichten gegenüber Betreiber und Betriebs- bzw. Personalrat.

4.2. Träger von Einrichtungen, in denen Tierversuche an Wirbeltieren durchgeführt werden, haben zudem gem. $\$ 8$ b TierSchG einen oder mehrere Tierschutzbeauftragte zu bestellen. Der Tierschutzbeauftragte unterscheidet sich vom gesetzgeberischen Leitbild der bislang erörterten Beauftragtenregelungen durch die Ausrichtung auf einen nicht-anthropozentrischen Bereich ${ }^{12}$; er hat über die Einhaltung der tierschutzrechtlichen Regelungen zu wachen und dient damit allein dem tierschützerischen Anliegen, ist also nicht für menschliche Belange des Gesundheits- oder im weiteren Sinne des Umweltschutzes zuständig. ${ }^{3}$ Neben der Überwachungsfunktion hat der Tierschutzbeauftragte innerbetrieblich auf die Vermeidung und Beschränkung von Tierversuchen hinzuwirken, zu jedem Genehmigungsantrag Stellung zu nehmen und die mit den Tierversuchen und mit der Haltung der Versuchstiere befaßten Personen zu beraten.

\section{Der Datenschutzbeauftragte}

Schließlich soll noch die Beauftragtenregelung des Datenschutzrechts Erwähnung finden. Nach der Vorschrift des $\$ 36$ I BDSG haben die nicht-öffentlichen Stellen, die personenbezogene Daten automatisch verarbeiten, einen Datenschutzbeauftragten zu bestellen. Neben natürlichen sowie juristischen Personen des Privatrechts kommen als Normadressaten auch die öffentlich-rechtlichen Wettbewerbsunternehmen nach $\$ 27$ BDSG in Betracht. Maßgebliches Kriterium für die Teilnahme am Wettbewerb ist das Anbieten von Leistungen, die auch von vergleichbaren privaten Unternehmen erbracht werden. ${ }^{4}$ Dies ist bei den durch privatrechtliche Behandlungsverträge erbrachten ärztlichen Leistungen kommunaler Krankenhäuser und Universitätskliniken der Fall.

\section{Das Beteiligungsverfabren}

Im folgenden gilt es, das der Bestellung und Abberufung zugrunde liegende Verfahren zu untersuchen. Das Personalvertretungsrecht kommt in dieser Frage zur Anwendung, soweit für den vorliegenden Bereich in den Personalvertretungsgesetzen ein entsprechender Beteiligungstatbestand enthalten ist. ${ }^{15}$

12 Zu diesem Begriff naher Caspar, Tierschutz unter rechtsethischem Aspekt, ARSP Juli 1995 (i. E.).

I3 Dies durfte den Gesetzgeber im Gegensatz zu den anderen Beauftragtenregelungen dazu bewogen haben, seine Stellung innerhalb der Forschungseinrichtung zumindest formell durch Bezugnahme auf dessen Weisungsfreiheit bei der Aufgabenerfullung in $\$ 8$ b VI TierSch G zu starken.

14 Vgl. dazu Ordemann/Schomerus, Kommentar zum Bundesdatenschutzgesetz, 5. Aufl., Munchen 1992, $\$ 27$ Rn.1.6., \$12 Rn.6., \$I8, Rn.6.

is Nach Art.75 I GG steht dem Bund fur die Rechtsverhaltnısse der Beschaftıgten im offentlichen Dienst von Landern, Gemeinden und anderen Korperschaften des offentlichen Rechts nur eine Rahmengesetz- 
Die Personalvertretungsgesetze sowohl des Bundes als auch der Länder enthalten in prozeduraler Hinsicht gestufte Formen der Beteiligung des Personalrats. ${ }^{16}$ Die stärkste Beteiligungsform ist die Mitbestimmung, die grundsätzlich eine übereinstimmende Willensbildung zwischen Dienststellenleiter und Personalvertretung erfordert und der Personalvertretung faktisch ein Vetorecht einräumt. Die Beteiligung geht zudem dort über den Akt der bloßen Zustimmung hinaus, wo der Personalvertretung in bestimmten Fällen der uneingeschränkten Mitbestimmung ein Initiativrecht zusteht, welches in das förmliche Mitbestimmungsverfahren übergeleitet wird. ${ }^{17}$

Unterhalb der Ebene der Zustimmung steht dem Personalrat in einigen Angelegenheiten ein Mitwirkungsrecht zu, wodurch der Personalvertretung ermöglicht wird, in Überlegungen und Entscheidungsprozesse über bestimmte Regelungen und Maßnahmen der Betriebsleitung unmittelbar einbezogen zu werden. Im Verfahren der Mitwirkung kann bei Einwendungen der Personalvertretung die Dienststelle letztlich auch gegen den Willen des Personalrats die von ihr intendierten Maßnahmen durchsetzen. Schließlich besteht als schwächste Form der Beteiligung nach den Personalvertretungsgesetzen noch das Recht der Anhörung.

\section{Zur Anwendbarkeit gesetzlicher Mitbestimmungstatbestände}

2.1. Nach den Personalvertretungsgesetzen sowohl des Bundes als auch der Länder hat der Personalrat über Personalangelegenheiten der Angestellten und Arbeiter bei der Einstellung mitzubestimmen. Die Bestellung einer zuvor betriebsfremden Person zum Betriebsbeauftragten kann somit die Mitbestimmungspflicht auslösen. Anknüpfungspunkt für die Beteiligung der Personalvertretung ist in diesem Fall jedoch ganz allgemein die Integration eines bislang neuen Arbeitnehmers in den Betrieb, unabhängig davon, ob dieser nun im Rahmen seiner zukünftigen Arbeit die Funktion des Betriebsbeauftragten wahrnehmen soll oder nicht. ${ }^{38}$

In der Regel wird der Betriebsbeauftragte hingegen aus dem Stab der bereits im Betrieb beschäftigten Personen bestimmt, so daß die Einstellungsklausel in der Praxis im Hinblick auf die Bestellung von Betriebsbeauftragten von untergeordneter Bedeutung ist. ${ }^{\text {" }}$

Eine mitbestimmungspflichtige Bestellung unter dem Aspekt der Übertragung einer höher zu bewertenden Tätigkeit kommt ebenfalls nicht in Betracht. Gemeint sind

gebungskompetenz zu. Fur die Beschaftigungsverhaltnısse der Bediensteten in staatlichen Krankenhausern und Unıversitatskliniken sind somit die Personalvertretungsgesetze der cinzelnen Bundeslander maßgeblich. Ausgenommen hiervon bleıben jedoch die Beschafugten in den von der Bundeswehr betriebenen Krankenhausern. Fur die zıvilen Beschaftigten der Bundeswehr gilt das BPersVG, fur die Soldaten in Dienststellen lehnt das BVerwG jedoch die Anwendung der Vorschriften des BPersVG ab; vgl. NVwZRR I 995 , S. 93 f.

I6 Auf Grund der strukturell weitgehenden Übereınstimmung zwischen Landes- und Bundespersonalvertretungsgesetz fur den hıer interessierenden Regelungsbereich rechtfertigt es sich, Im folgenden den Schwerpunkt der Ausfuhrungen auf die fur Bundesbedienstete geltenden Bestimmungen des Bundespersonalvertretungsgesetzes zu legen.

17 Ausfuhrlich zu den Formen der Mitbestimmung, Sollner/Reinert, Personalvertretungsrecht, Baden-Baden 1985, S. $160 \mathrm{ff}$

$18 \mathrm{Vgl}$. Lorenzen/Haas/Schmıdt, Bundespersonalvertretungsgesetz, Kommentar, Loseblattsammlung, $\$ 75$, Rn. I6; sowie Bahrle, Die arbertsrechtliche Stellung der Umweltschutzbeauftragten, UPR 1995, S. 94 fur den Bereich des BetrVG.

19 Zumeist - insbesondere in kleinen oder mıttleren Betrıeben - wird die Tatigkeit des Betriebsbeauftragten nebenberuflich ausgeubt, vgl. etwa Rehbinder (Fn. 3) s988, S. 123 ; fur den Datenschutzbeauftragten, Herb, Zur Kundbarkeit des betrieblichen Datenschutzbeauftragten, S. 60, in: Ehmann (Hrsg.), Der Datenschutzbeauftragte im Unternehmen, Koln I 993. 
damit ausschließlich Maßnahmen, die sich auf das Arbeitsentgelt auswirken. ${ }^{20} \mathrm{Da}$ die Bestimmung eines Beschäftigten zum Betriebsbeauftragten in der Regel keinen Einfluß auf dessen Zuordnung zu einer tariflichen Lohn- oder Gehaltsgruppe hat, kommt der zuvor erörterte Mitbestimmungstatbestand nicht zur Anwendung.

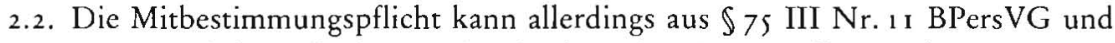
den im wesentlichen gleichlautenden landesrechtlichen Parallelvorschriften folgen, nach welchen der Personalrat über Maßnahmen zur Verhütung von Dienst- und Arbeitsunfällen und sonstigen Gesundheitsschäden mitzubestimmen hat. ${ }^{2 t}$

Die tatbestandlichen Voraussetzungen der Mitbestimmungsvorschrift liegen hinsichtlich des arbeitsschutzrechtlichen Betriebsbeauftragten nach RVO und ASiG ohne weiteres vor, dient der danach zu bestellende Betriebsbeauftragte doch gerade dem Schutz der Beschäftigten vor arbeitstypischen Gefahren. Seine Benennung stellt damit eine Maßnahme zur Verhütung von Arbeitsunfällen i.S. der Mitbestimmungsnorm dar. ${ }^{22}$

Dagegen kommt eine Mitbestimmung bei der Bestellung des Datenschutzbeauftrag$\operatorname{ten}^{23}$ sowie des Tierschutzbeauftragten über diese Vorschriften nicht in Betracht. Weder werden diese tätig, um Arbeitsunfälle zu verhindern, noch gehört es zu ihren Aufgaben, ganz allgemein Schädigungen der menschlichen Gesundheit präventiv zu begegnen. Datenschutzgesetz und Tierschutzgesetz haben dies nicht einmal mittelbar zum Ziel. ${ }^{24}$

Anders verhält es sich hingegen mit den medizintypischen Beauftragtenregelungen innerhalb der Strahlenschutz- und RöntgenVO sowie des MedProdGesetzes, die allgemein der Verhütung von Gesundheitsschäden bei Personal und Dritten dienen. Dabei kann hier offen bleiben, ob unter Maßnahmen zur Verhütung von Unfall- und Gesundheitsschäden überhaupt Vorkehrungen zum Schutz betriebsexterner Personen fallen. ${ }^{25}$ Selbst wenn man den Anwendungsbereich dieser Mitbestimmungsnorm exklusiv nur auf die Bediensteten eines Betriebes beschränkt wissen will, so dienen die hier erörterten medizintechnischen Vorschriften neben dem Schutz der Patienten zumindest auch den einzelnen Arbeitnehmern und fallen in den Anwendungsbereich der Mitbestimmungsnorm. Die RöVO wie auch die StrahıSchVO enthalten nämlich eigens Vorschriften für beruflich besonders strahlenexponierte Personen. ${ }^{26}$

Schließlich umreißt das Medizinproduktegesetz den Kreis der zu schützenden Personen in der Eingangsbestimmung derart extensiv, daß nicht nur Patienten, sondern auch Anwender und darüber hinausgehend auch ganz allgemein dritte Personen in

20 Lorenzen/Haas/Schmitt (Fn. 18), \$75 I Nr. 2 BPersVG Rn. $26 \mathrm{ff}$.

21 So etwa $\$ 72$ IV Nr. 7 NWPersVerG; $\$ 75$ III Nr. 13 Nds.PersVG; $\$ 86$ I Nr. 16 Hamb.PersVerG mit ausdrucklıcher Erwahnung auch von Maßnahmen zur Verhutung von Berufskrankheiten; $\$ 79$ I Nr. 8 BaWÜPersVerG; $\$ 74$ III Nr. I I ThurPersVG; \$75 IV Nr. 8 BayPersVG; \$74 I Nr. 6 HessPersVG.

22 In Hessen ordnet $\$ 74$ I Nr. 3 PersVG ausdrucklich an, daß furr die Bestellung und Abberufung von Sicherheitsbeauftragten und Fachkraften fur Arbeitssicherheit ebenso wie von Datenschutzbeauftragten und Frauenbeauftragten der Personalrat mitzubestimmen hat. Ob diese landesrechtliche Vorschrift im Hinblick auf die Sicherhettsbeauftragten mit \$719 RVO, der der Personalvertretung ledıglich ein Mitwirkungsrecht einraumt, ubereinstimmt, ist zumindest sehr fraglich; vgl. insbesondere auch zu Art. 31 GG, Hoffmann, Normenkonkurrenzen im Öffentlichen Recht, JA 1981 , S. 16 I ff.

23 Nach BVerwG PersV 1987, 287 = NJW 1987, S. 16 8 8, wo es um die Frage der Reinigungshaufigkeit von Diensträumen ging, muß die Maßnahme in \$75 III Nr. II BPersVG maßgeblich darauf abzzelen, das Risiko von Gesundheitsschadigungen oder Unfallen zu verringern oder einen effektiven Arbeits- und Gesundheitsschutz zu gewährleisten.

${ }_{24}$ Allenfalls erwagenswert als Quelle moglicher gesundheitlicher Gefahren ist in diesem Zusammenhang die qua Legaldefinition als Tierversuch enngestufte gentechnische Manipulation am Erbgut von Tieren. Dieser Gefahrdungslage tragen jedoch bereits die spezielleren Vorschriften des Gentechnikgesetzes Rechnung, nach denen die Bestellung eines fur die betriebliche Sicherhert zustandigen Sicherheitsbeauftragten obligatorisch ist.

25 Mit dieser Tendenz BVerwG NJW 1987, S. 1658.

$26 \mathrm{Vgl} . \$ 44$ sowie $\$ \$_{49} \mathrm{ff}$. StrahlSchVO; $\$ 3 \mathrm{Iff}$. RoVO. 
den gesetzlichen Schutzbereich dieses Gesetzes einbezogen werden. ${ }^{27}$ Die Bestellung und Abberufung von Betriebsbeauftragten im medizintechnischen Bereich stellt also durchweg eine Maßnahme des vorbeugenden Gesundheitsschutzes dar.

Der Abfallbeauftragte ist dagegen leitmotivisch weniger zur Prävention von Gesundheitsschäden denn als genuin umweltrechtliches Instrument zur Vermeidung und fachkompetenten Entsorgung von Abfällen innerhalb eines Betriebes konzipiert. Dennoch zeigt sich gerade an der Art der im Bereich von Krankenhäusern anfallenden Abfälle, daß eine ordnungsgemäße Abfallentsorgung nicht zuletzt auch im Interesse der Gesundheit der Beschäftigten liegt. Nur am Rande sei vermerkt, daß sich frühe Ansätze des Abfallrechts historisch aus der allgemeinen Gesundheitsvorsorge entwickelt haben und zunächst der Ausbreitung von lebensbedrohenden Infektionskrankheiten wie z. B. der Pest und der Cholera entgegenwirken sollten. In den modernen Industriegesellschaften wird dieser Aspekt durch das Problem der quantitativen Abfallerzeugung mittlerweile eher in den Hintergrund gedrängt. ${ }^{28}$

Außer für den Tierschutz- und Datenschutzbeauftragten bezieht somit der tatbestandliche Anwendungsbereich des $\$ 7$ s III Nr. I I BPersVG und der entsprechenden Landespersonalgesetze die Bestellung und Abberufung von Betriebsbeauftragten ein.

\section{Die Subsidiarität der gesetzlichen Mitbestimmungsregeln}

In $\$ 75$ III BPersVG und den entsprechenden landesrechtlichen Vorschriften steht die Mitbestimmung des Personalrats jedoch unter dem Vorbehalt, daß keine andere gesetzliche oder tarifliche Regelung eingreift. Der Ausschlußtatbestand liegt also vor, wenn ein Sachverhalt unmittelbar durch Gesetz geregelt ist, dergestalt, daß es zum Vollzug der Regelung keines besonderen Ausführungsaktes bedarf. ${ }^{29}$ Erfolgt demnach eine verfahrensmäßige Regelung der Bestellung des Personalrats bereits in besonderen Fachgesetzen, so kommt ein Rückgriff auf das Personalvertretungsgesetz nicht in Betracht. Unter gesetzlicher Regelung hat man in diesem Zusammenhang jedes durch Gesetz oder auf Grund eines Gesetzes geschaffene Recht zu verstehen, so daß hierunter auch Rechtsverordnungen fallen. ${ }^{30}$

3.I. Die Frage der Beteiligung bei der Bestellung des Betriebsbeauftragten wird sowohl vom ASiG als auch von der RVO geregelt. \$719 I S. 2 RVO bestimmt wörtlich: "Die Bestellung hat unter Mitwirkung des Betriebsrats (Personalrats) zu erfolgen.« Eine die Zustimmung der Personalvertretung erfordernde, also eine über das bloße Mitwirkungsverfahren hinausgehende Beteiligung wird dagegen von der entsprechenden Beauftragtenregelung in $\$ 9$ III ASiG angeordnet. Danach sind die Fachkräfte für Arbeitssicherheit mit Zustimmung des Betriebsrats zu bestellen und abzuberufen. Über $\$$ I $6 \mathrm{ASiG}$ gilt dies auch für den medizinischen und sicherheitstechnischen Arbeitsschutz in Verwaltungen und Betrieben des öffentlichen Dienstes. Es besteht daher Einigkeit darüber, daß die Maßnahmen ebenso der Mitbestimmung der Personalvertretung unterliegen, mit der Folge, daß der Personalrat ausdrücklich der Benennung zustimmen muß.31

\footnotetext{
27 Vgl. \$I MedProdG.

28 Zur Abfallbeseitigung Im Mittelalter vgl. Heine, Okologie und Recht - Zur historischen Entwicklung normativen Umweltschutzes, GA 1989, S. 123.

29 BVerwG, PersR 1986, S. 178.

30 So OVG Hamburg, PersV 1985,33 .

31 In diesem Sinne etwa Lorenzen/Haas/Schmitt (Fn. 18) \$75 BPersVG Rn. 175; Grabendorff/Windscheid/Illbertz/Widmaier, Bundespersonalvertretungsgesetz, 7. Aufl., Stuttgart 1991, $\$ 75$, Rn. 157; etwa auch Krieg/Orth/Welkoborsky, Landespersonalvertretungsgesetz für Nordrhein-Westfalen, 4. Auf!. Koln 1987, S. 429 f.; mit Einschrankungen allerdings Dietz/Ruchardi, Bundespersonalvertretungsgesetz, 2. Aufl., Munchen $1978, \$ 75$ Rn. 425 , wonach dennoch ein Funktionszusammenhang zu dem in Nr. 1 I umschriebenen Mitbestimmungstatbestand angenommen wird.
} 
Daneben erfolgt eine explizite mitbestimmungsrelevante Erwähnung des Personalrats lediglich im Gentechnikgesetz. Dort besteht nach \$16 I GenTSVO ein Anhörungsrecht der Pesonalvertretung bei der Bestellung des Sicherheitsbeauftragten.

3.2. Darüber hinaus fehlen spezialgesetzliche Beteiligungsvorschriften. Die Strahlenschutzverordnung fordert in $\$ 29$ III S. 3 StrahlSchVO zwar, daß eine Abschrift der bei Bestellung und Abberufung des Strahlenschutzbeauftragten gesetzlich geforderten behördlichen Anzeige auch dem Betriebs- bzw. Personalrat auszuhändigen ist. Damit wird jedoch der Beteiligungslevel einer Anhörung deutlich unterschritten $^{32}$, zumal eine Anzeige die Personalvertretung lediglich nachträglich über eine veränderte Tatsachenlage in Kenntnis setzt und ihr verwehrt ist, der Betriebsleitung vorab die eigene Auffassung darzutun.

Ein Rückgriff auf die mitbestimmungsrechtlichen Vorschriften kommt somit nur in Betracht, wenn das Fehlen verfahrensmäßiger Bestimmungen hinsichtlich Bestellung bzw. Abberufung des Betriebsbeauftragten innerhalb der Fachgesetze nicht implizit als Ausschluß jeglicher Mitbestimmung zu werten ist. Insoweit muß geklärt werden, ob dem gesetzgeberischen Schweigen ein Erklärungsgehalt zukommt oder ob in den einschlägigen Fachgesetzen das Verfahren zur Abberufung und Bestellung der Betriebsbeauftragten offengelassen wurde - etwa weil der Gesetzgeber diese Frage nicht hatte regeln wollen oder die Regelungsbedürftigkeit schlicht verkannt hat. Ein historischer Rückblick Jäßt erkennen, daß bereits recht früh erwogen wurde, dem Betriebsrat ein Mitbestimmungsrecht bei der Bestellung des Immissionsschutzbeauftragten einzuräumen ${ }^{33}$ und in der Folgezeit auch die Beteiligung des Betriebsrats an der Benennung des Betriebsbeauftragten im Gesetzgebungsverfahren zum Bundesdatenschutzgesetz in Betracht gezogen wurde. ${ }^{34} \mathrm{Daß}$ der Gesetzgeber die mitbestimmungsrechtliche Fragestellung sehr wohl erkannt hat und es ihm um die Festschreibung einer minimalen Beteiligungsebene der Arbeitnehmervertretung gegangen ist, belegen auch die vielfältigen Bezugnahmen auf den Personal- bzw. Betriebsrat innerhalb der einschlägigen Fachgesetze. So findet sich die bereits angesprochene Anzeigepflicht des Betriebsleiters gegenüber dem Personalrat wie auch die Kooperationspflicht in der früheren StrahlSchVO.35

Für einen mitbestimmungsfreien Vorgang spricht schließlich auch, daß die frühen Vorschriften über den arbeitsschutzrechtlichen Betriebsbeauftragten in der RVO und dem ASiG bereits Mitbestimmungs- bzw. Mitwirkungsrechte für den Personalrat enthielten, so daß davon ausgegangen werden muß, daß der Gesetzgeber bei entsprechendem politischen Willen derartige Bestimmungen in die später erlassenen Fachgesetze inkorporiert hätte. ${ }^{36}$ Wenn der Gesetzgeber eine weitergehende Mitbestimmung tatsächlich wünschte, ist zu vermuten, daß er dies dann auch explizit zum Ausdruck gebracht haben würde. Sein Schweigen muß daher in dem Sinne verstanden werden, daß über die genannten Verpflichtungen zur Kooperation und zur Anzeige der Abberufung keinerlei partizipatorische Einflußnahme stattfinden sollte, mithin die verschiedenen Beauftragten allein durch die Betriebsleitung ernannt und entlassen werden sollten. Ein Rückgriff auf die Mitbestimmungsnorm des Bundes und der Länder hinsichtlich der Verhütung von Arbeits- und Gesundheitsschäden

32 Ungenau hier der Entwurf des Umweltgesetzbuches AT, Fn. 1, wonach fur den Strahlenschutzbeauftragten bereits nach der bisherigen Regelung ein Anhorungsrecht bei dessen Abberufung bestehe, S. 391 .

33 Roth, Fn. 10, S. 90 mit Hinwers auf Beratungen des Innenausschusses des Bundestags.

34 Damals wurde anlaßlıch einer offentlichen Anhorung vor dem Innenausschuß von Gewerkschaftsseite die Forderung nach Betelligung des Betriebsrats bei der Bestellung des Datenschutzbeauftragten erhoben. Darauf weist Weber, Der Betrrebsbeauftragte, Berlin 1988, S. 86 hın.

$35 \$ 30$ II StrahlSchVO, BGBl. 1976 I, S. 2905 .

36 Ahnlıch auch Weber a. a. O., der bemangelt, daß dieser Fragenkomplex in der umweltschutzrechtlıchen Literatur bislang nicht angesprochen worden ist. 
kann daher auf Grund der Vorrangklausel der Personalyertretungsgesetze nicht erfolgen.

\section{Der Betriebsbeauftragte im Spannungsfeld zwischen Umweltschutz und betrieblichen Interessengegensätzen}

Das vorliegende Ergebnis gibt dazu Anlaß, die mitbestimmungsrechtliche Fragestellung unter rechtspolitischen Aspekten zu reflektieren und yor dem Hintergrund gegenwärtiger Vereinheitlichungsbemühungen im Umweltrecht Erwägungen über eine Neugestaltung der Figur des Betriebsbeauftragten anzustellen. In diesem $\mathrm{Zu}-$ sammenhang kann am Rande insbesondere auf die Entwürfe zum Allgemeinen und Besonderen Teil eines Umweltgesetzbuches und die dort zugrunde liegende Konzeption des Betriebsbeauftragten eingegangen werden.

In der Diskussion über eine Neuakzentuierung der Rechtsstellung des Betriebsbeauftragten lassen sich als konträre Lager leicht die Vertreter der Arbeitnehmer auf der einen und die der Arbeitgeber auf der anderen Seite ausmachen. Es verwundert deshalb nicht, wenn vor allem die Frage nach der Mitbestimmung bei Bestellung und Abberufung dem ideologisch geprägten Vorverständnis folgt: Wird von gewerkschaftlicher Seite die Forderung nach einer Stärkung der arbeitsrechtlichen Stellung des Betriebsbeauftragten erhoben, so betont die arbeitgeberfreundliche Seite die Kontraproduktivität eines derartigen Ansinnens. ${ }^{37}$

Neben diesen beiden Lagern ist noch eine dritte Position auszumachen, die sich mit dem Betriebsbeauftragten insbesondere unter umweltrechtlichem Aspekt auseinandersetzt, dem reformerischen Bemühen grundsätzlich offen gegenübersteht, in der Frage der Mitbestimmung des Personal- bzw. Betriebsrats sich jedoch eher zurückhält. ${ }^{38}$ Die Ablehnung einer Mitbestimmung erfolgt dort nicht primär aus arbeitsrechtlichen Gründen, sondern sie wird mit dem Leitbild des Betriebsbeauftragten als einer innerbetrieblichen Kontrollinstanz begründet. Eine kompetenzielle Unabhängigkeit von der Betriebsleitung, so die Argumentation, würde Sinn und Zweck der Konzeption selbst konterkarieren, zumal die Vorteile freiwilliger Selbstregulierung dadurch zunichte gemacht würden. Die weitere Verrechtlichung seiner lediglich formalen Verhandlungsmacht durch eine Einbindung des Beauftragtenmodells in das System der arbeitnehmerischen Mitbestimmung sei insoweit dysfunktional, man begebe sich »von der Szylla der Ineffizienz in die Charybdis der Überlegalisierung «.,39 Dies wird erklärt mit dem zumindest partiellen Scheitern regulativer Politik samt der daraus resultierenden interventionistischen Rechtsprogrammatik. $4^{\circ}$

Aber kann eine Erweiterung der Mitbestimmung der Belegschaft das Konzept freiwilliger Selbstkontrolle wirklich ernsthaft in Frage stellen? Ursprünglich diente die Beauftragtenregelung doch nur einer eigeninitiierten Selbstkontrolle, für die gerade das Fernbleiben des staatlichen Zwangs motivierend wirken sollte. Eine innerbetriebliche Demokratisierung dieses Aufgabenbereichs würde hieran nichts ändern; im Gegenteil: Das konsensuale Verfahren bei personellen Entscheidungen über den Betriebsbeauftragten ließe einen positiven Feedback-Effekt zwischen Arbeitgeber-

37 Vgl. ennerseits IG Metall, Vorstand (Hrsg.), Eckpunkte für eıne Betriebsvereinbarung zum Umweltschutz in Unternehmen, S. I; andererseits aus der Sicht der Arbeitgeberseite, Sander (Fn. 10), S. 51; sowie im Ergebnis Steiner (Fn. 4), S. I14I; zu weiteren Nachwersen s. Renken, Der Betriebsbeauftragte fur Umweltschutz, KJ 1994, S. 224 ff.

38 So etwa grundsatzlich Kloepfer/Rehbınder/Schmıdt-Aßmann, Umweltgesetzbuch. Allgemeiner Teil, wonach eine Verstarkung der Rechtsstellung des Betriebsbeauftragten durch Annaherung an das Modell des Strahlenschutzbeauftragten erfolgen soll, S. ${ }_{3} 84$ ff., zogeriıcher Rehbinder (Fn. I) 1988, S. 125 .

39 Rehbinder (Fn. I), S. 124 .

$40 \mathrm{Zu}$ dem Begriff des regulatorischen Trilemmas in diesem Sinne Rehbinder (Fn. I), S. I Io. 
seite und den im Betrieb Beschäftigten erwarten, der letztlich zu einer Stärkung der Akzeptanz des Betriebsbeauftragten und damit zur Effektuierung seiner Arbeit führen würde. Der Betriebsbeauftragte erschiene dann tatsächlich weniger als Statthalter des Arbeitgebers denn als Mann des Betriebes mit klaren Interessenvertretungsansprüchen und -befugnissen für die gesamte Belegschaft.

Auch die Argumentation einer grundsätzlichen Inkompatibilität von Ökologie und Ökonomie kann nicht überzeugen. Daß der Personal- bzw. Betriebsrat nicht als "geborener Hüter des Umweltschutzes «"1 innerhab des Betriebs anzusehen sein dürfte, spricht nicht gegen eine stärkere Demokratisierung dieser Institution. Ist etwa umgekehrt der den Betriebsbeauftragten bestellende Arbeitgeber natürlicher Garant des Umweltschutzes?

Zumindest gibt es eine essentielle Verbindung zwischen dem Betriebsbeauftragten und der Arbeitnehmervertretung, die den Bereich der gesundheitspräventiven Maßnahmen betrifft und für die sich keine Entsprechung im Verhältnis des Arbeitgebers zum Betriebsbeauftragten finden läßt. Mag das Ziel modernen Umweltschutzes weit über den bloßen arbeitsrechlichen Gesundheitsschutz hinausgehen - es zeigt sich dennoch ein wichtiger Berührungspunkt in der Gestaltung der Arbeitswelt und dem Anliegen des Umweltschutzes, der trotz aller prinzipiell bestehenden Aporien zwischen betrieblicher Mitbestimmung und ökologischen Erwägungen nicht geleugnet werden kann. Hierin ist denn auch ein wichtiges Versäumnis bisheriger Umweltpolitik und aller konkreten reformerischen Bemühungen angesprochen: daß faktisch eine Ausklammerung umweltrechtlicher Fragestellungen von den ihnen zugrundeliegenden sozialökonomischen Faktoren stattfindet, indem den ökologischen Basisprinzipien wie der Intensivierung von Öffentlichkeit, der Transparenz von Entscheidungsprozessen und der Optimierung von Selbstrepräsentanz Betroffener keine oder zumindest nur eine zu geringe Rolle beigemessen wird.

Dies macht exemplarisch gerade die hier diskutierte Frage deutlich. Sieht man den Betrieb als ein teilautonom-offenes System sich selbst regulierender arbeitsweltlicher Beziehungen, so sind keine rationalen Gründe ersichtlich, warum man den Betriebsbeauftragten im Umweltrecht intern allein der Betriebsleitung unterstellen sollte.

Ein Mitspracherecht des Personal- bzw. Betriebsrats in diesen Fragen darf freilich andererseits nicht zu übertriebenen Hoffnungen auf die Einwirkungsmöglichkeiten des Betriebsbeauftragten verleiten. Dennoch ist vorstellbar, daß das beklagte Manko an Durchsetzungsfähigkeit ${ }^{2}$ des Betriebsbeauftragten konkret auch durch eine Verbesserung des arbeitsrechtlichen Status abgebaut werden könnte, was das in einigen Beauftragtenregelungen bereits enthaltene allgemeine Benachteiligungsverbot ${ }^{43}$ sinnvoll ergänzen würde. Während der zuvor erörterte Bereich lediglich konstitutive Entscheidungen hinsichtlich der Beauftragtenregelung - nämlich Einsetzung und Absetzung eines Arbeitnehmers - bedarf, ist damit auch eine Verbesserung des individuellen Beschäftigungsverhältnisses angesprochen.

Zumindest im Immissionsschutzgesetz wird vom Gesetzgeber eine arbeitsrechtliche Stärkung der Position des Betriebsbeauftragten vorgenommen: Nach dem anläßlich einer Novellierung des BImSchG eingefügten Absatz II des $\$ 58$ BImSchG ist die Kündigung des Arbeitsverhältnisses eines zum Betriebsbeauftragten bestellten Arbeitnehmers durch den Betreiber unzulässig, es sei denn, daß Tatsachen vorliegen, die den Betreiber zur Kündigung aus wichtigem Grund ohne Einhaltung einer Kün-

41 So aber Steiner (Fn. 4), S. 1141 ; ahnlich Weber (Fn. 34), S. 87

42 Rehbinder (Fn. 1), 1988, S. 123 ; Renken (Fn. 37), S. $228 \mathrm{f}$

43 So etwa $₫$ i4 IV RovO; $₫ 30$ IV StrahlSchVO; i f $A b f G ; ~ \$ 8$ b VI TierSchG. 
digungsfrist berechtigen. Entsprechendes gilt für den Zeitraum eines Jahres nach dessen Abberufung als Immissionsschutzbeauftragten. Obwohl mit dieser Regelung die Kündigung nicht an die Zustimmung der Arbeitnehmervertretung gekoppelt ist, wie dies $\$ 47$ BPersVG für die außerordentliche und $\$ \$$ i $5, x 6 \mathrm{KSchG}$ für die ordentliche Kündigung vorsehen, wird dennoch ein besonderer Kündigungsschutz gewährt, und zwar mit der amtlichen Begründung, der Immissionsschutzbeauftragte solle nicht aus Furcht vor Entlassung an einer wirksamen Wahrnehmung seiner Aufgaben gehindert werden. ${ }^{44}$ Hinter dieser an den Kündigungsschutz für Betriebs- und Personalräte angelehnten Regelung des Immissionsschutzgesetzes bleiben die Vorschläge zur Kodifikation des Umweltrechts jedoch zurück.

Trotz eines grundsätzlichen Bekenntnisses für eine stärkere Anbindung des Betriebsbeauftragten an die Belegschaft, die den vielfältigen Verknüpfungen von Umweltund Arbeitsschutz Rechnung tragen würde, wird ein an den Kündigungsschutz von Personal- bzw. Betriebsratsmitgliedern ausgerichteter Kündigungsschutz abgelehnt. Statt dessen soll nach $\$ 98$ I des UGB AT der Personalvertretung lediglich ein Anhörungsrecht bei der Abberufung des Betriebsbeauftragten eingeräumt werden. Eine die arbeitsrechtliche Stellung des Betriebsbeauftragten sichernde Regelung, die das allgemeine Benachteiligungsverbot des $₫ \mathrm{roz}_{2}$ UGB AT flankieren könnte, fehlt jedoch. Zudem verzichtet der Reformvorschlag auf eine Belegschaftsbeteiligung bei der Bestellung völlig. Auch der Gesetzesentwurf vom UGB BT setzt innerhalb der nach wie vor beibehaltenen besonderen Beauftragtenregelungen keine weitergehenden Akzente. ${ }^{45}$

Den vielfältigen Berührungspunkten zwischen Arbeitsschutz und Umweltschutz wird damit - trotz gegenteiliger Bekundungen - im Ergebnis vom Entwurf nicht Rechnung getragen. Wie gezeigt, zielen die umweltrechtlichen Vorschriften größtenteils auch auf Belange des Arbeits- und Gesundheitsschutzes und betreffen damit einen traditionell mitbestimmungsrechtlich geprägten Bereich. ${ }^{16}$ Dennoch wird weiterhin an dem patriarchalischen Beauftragtenkonzept festgehalten. Möglicherweise soll damit eine Verstimmung auf Seiten der Arbeitgeber verhindert werden. So lassen die gegenwärtigen Reformvorschläge die Erkenntnis, daß eine moderne Form der Selbstkontrolle, wie sie der Idee nach dem Rechtsinstitut des Betriebsbeauftragten zugrundeliegt, nicht nur dem Applaus von oben, sondern in ganz entscheidendem Maße auch der Akzeptanz von unten bedarf, leider vermissen.

Die Einrichtung der Institution des Betriebsbeauftragten als eine Erscheinungsform des Konzepts der »regulierten Selbstregulierung « 47 im Umweltrecht hat sich bislang - sieht man einmal von überzogenen Erwartungen ab - grundsätzlich bewährt. Wenn nunmehr Gelegenheit zu einer Weiterentwicklung dieses Mediums indirekter Steuerung besteht, sollte es in Richtung auf ein mitbestimmungsoptimiertes Konzept genutzt werden. Den Zielkonflikt zwischen Ökologie und Ökonomie kann man nicht lösen, indem man ihn immer wieder heraufbeschwört. Dazu gehört vielmehr die Bereitschaft, innovative Ansätze auch wirklich durchsetzen zu wollen.

44 BT-Drucks. 11/4909, S. 25

$45 \mathrm{Vgl}$. die Regelungen uber den Umweltbeauftragten fur Strahlenschurz nach $\$ \$ 400 \mathrm{ff}$. mit Verweıs auf die vor die Klammer gezogenen Vorschriften des UGB-AT sowıe die Vorschriften uber den Abfallbeauftragten in $\$ \$ 82$ und den Gewasserschutzbeauftragten in $\$ \$ 269$ f.; allein im immıssionsschutzrechtlıchen Teil bleibt es bei der Regelung de lege lata, wobei in der Kommentierung zu $\$ 352$ BT jedoch auf die Moglichkeit einer Ubernahme dieser Regelung in den AT hingewıesen wird, Jarras, Kloepfer, Kunig u. a. Entwurf zum Umweltgesetzbuch BT, Berlin 1994, S.692.

46 Weber (Fn. 34), S. 89 .

$47 \mathrm{Zu}$ diesem Begriff Hoffmann-Riem, Ökologisch orientiertes Verwaltungsrecht - Vorklärungen, AoR 1994, S. $608 \mathrm{ff}$. 